JOURNAL OF

SYNCHROTRON

RADIATION

ISSN 1600-5775

Received 21 March 2016

Accepted 17 October 2016

Edited by D. A. Reis, SLAC National Accelerator Laboratory, USA

Keywords: X-ray absorption tomography; diffraction scattering tomography; high pressure; basalt glass; $\mathrm{C}_{60}$ polymerization.

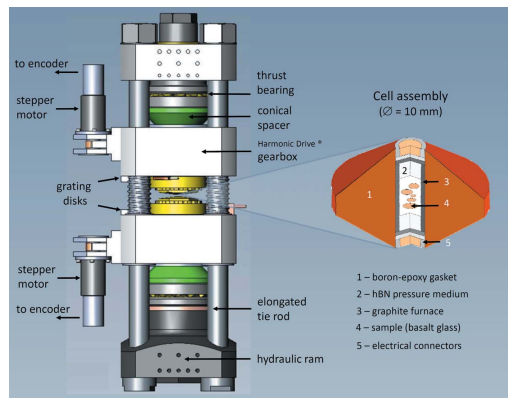

OPEN $\odot$ ACCESS

\section{Development of synchrotron X-ray micro-tomo- graphy under extreme conditions of pressure and temperature}

\author{
M. Álvarez-Murga, ${ }^{\mathrm{a}, \mathrm{b}}$ J. P. Perrillat, ${ }^{\mathrm{c} *}$ Y. Le Godec, ${ }^{\mathrm{d}}$ F. Bergame, ${ }^{\mathrm{d}}$ J. Philippe, ${ }^{\mathrm{d}}$ \\ A. King, ${ }^{e}$ N. Guignot, ${ }^{e}$ M. Mezouar ${ }^{a}$ and J. L. Hodeau ${ }^{b}$
}

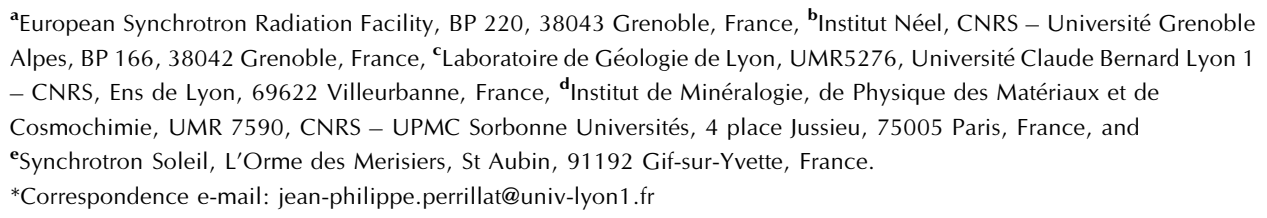

$\mathrm{X}$-ray tomography is a non-destructive three-dimensional imaging/microanalysis technique selective to a wide range of properties such as density, chemical composition, chemical states and crystallographic structure with extremely high sensitivity and spatial resolution. Here the development of in situ high-pressure high-temperature micro-tomography using a rotating module for the ParisEdinburgh cell combined with synchrotron radiation is described. By rotating the sample chamber by $360^{\circ}$, the limited angular aperture of ordinary highpressure cells is surmounted. Such a non-destructive high-resolution probe provides three-dimensional insight on the morphological and structural evolution of crystalline as well as amorphous phases during high pressure and temperature treatment. To demonstrate the potentials of this new experimental technique the compression behavior of a basalt glass is investigated by X-ray absorption tomography, and diffraction/scattering tomography imaging of the structural changes during the polymerization of $\mathrm{C}_{60}$ molecules under pressure is performed. Small size and weight of the loading frame and rotating module means that this apparatus is portable, and can be readily installed on most synchrotron facilities to take advantage of the diversity of three-dimensional imaging techniques available at beamlines. This experimental breakthrough should open new ways for in situ imaging of materials under extreme pressuretemperature-stress conditions, impacting diverse areas in physics, chemistry, geology or materials sciences.

\section{Introduction}

$\mathrm{X}$-ray tomography refers to the cross-sectional imaging of the interior of bulk objects through the use of an intense beam of high-energy X-ray photons. As it passes through the sample, the radiation is affected by photoelectric interactions and scattering. Several aspects of theses interactions can be mapped in three dimensions: the material-specific absorption of photons, spatial variations in the refractive index, the particles' size and shape, the crystallographic structure, the distribution of elements, and the local bonding structure. The most common form of tomography is X-ray absorption computed tomography $(\mu \mathrm{CT})$ whereby an intense hard X-ray beam is used to illuminate an object at many different angles of rotation producing a large number of two-dimensional radiographs. These radiographs are then computed to produce a three-dimensional (3D) image in which each volume element, or voxel, is assigned a grayscale that correlates with 
the local differences in mass density and mean atomic number. Initially developed for clinical diagnosis in medicine, the method has been extensively used in engineering and material sciences to investigate microstructures and damage mechanisms [see Banhart (2008) for a review]. The advent of highbrilliance, high-energy and spatially coherent synchrotron $\mathrm{X}$-ray beams has enhanced the spatial resolution down to the sub-micrometer scale, and continuous development of highefficiency fast detectors and algorithms lead to reductions in the time needed to record a complete CT scan, which is now in the sub-second range. These high spatial and temporal resolutions provide an opportunity to perform near real-time fourdimensional imaging for the characterization of dynamic phenomena in biology, geosciences and materials science. Another approach to CT imaging is to use the scattered radiation to form an image of the object. The distribution of elements can be mapped from spatial differences in X-ray fluorescence (e.g. Schroer, 2001), while spectroscopy contrast provides information on the local chemical speciation (e.g. Rau et al., 2003). The diffraction/scattering X-ray diffraction tomography (DSCT) is sensitive to the local crystalline state given by the $2 \theta$ ( $d$-spacing) contrast (e.g. Poulsen et al., 2004). It gives access to micrometer-scale $3 \mathrm{D}$ phase-selective maps and allows the retrieval of local structure information such as crystallite size, micro-strain, preferential orientations and defects. This technique is particularly appropriate for the characterization of complex multi-phase and multi-scale materials that require the discrimination of crystalline and amorphous phases within an inhomogeneous matrix.

Increasingly, X-ray tomography is being used to follow the evolution of samples under controlled environmental conditions. Coupled to a tensile/compression stage, micro-tomography enables the evolution of damage and microstructure to be followed in response to mechanical stress on materials as diverse as metallic alloys, foams, ceramics and ice (e.g. Salvo et al., 2003). Similarly, the effect of non-ambient temperature can be investigated by surrounding the sample with X-ray transparent resistive furnaces for heating or cryostats for cooling. Examples of application include the study of thermal treatment of ceramics and catalyst thermal sintering (Bernard et al., 2005), or bubble growth and phase separation in molten silicate glasses (Baker et al., 2012). So far, micro-tomography studies under high pressure remain limited to a few examples based in attenuation imaging. The main limitation for these kinds of studies is that the typical side opening of high-pressure cells is $<180^{\circ}$, which is not suitable for acquiring a complete set of projections. A compact panoramic highpressure apparatus with a $160^{\circ}$ opening has been designed at SPring-8 for tomography applications (Urakawa et al., 2010), but image reconstruction shows artifacts originating from shadow regions in the radiographs. Efforts have also been made to perform nano-imaging in a diamond-anvil cell using $\mathrm{X}$-ray transparent beryllium gaskets, and iterative methods to overcome the 'limited projections problem' (Liu et al., 2008; Wang et al., 2012; Lin et al., 2013). In practice, only GSECARS at the Advanced Photon Source at Argonne National Laboratory has the capability of performing absorption-based tomography in large-volume samples under high pressure $(\sim 10 \mathrm{GPa})$, high temperature $(\sim 1500 \mathrm{~K})$ and deformation, using a modified Drickamer cell allowing the full rotation of the sample chamber under load (Wang et al., 2005; Yu et al., 2016). This device has been successfully used to study the volumetric properties of non-crystalline materials (Lesher et al., 2009), and the deformation behavior of multi-phase composites under shearing (Wang et al., 2011). Nevertheless, this tomography module is installed within a hydraulic press frame on a dedicated beamlime which limits the application to other 3D imaging techniques from the scattering signal.

Herein, we report the development of a portable highpressure rotating cell for tomography measurements at synchrotron sources. Based on the Paris-Edinburgh apparatus, it enables independent and controlled rotation of each opposed anvil for a full $360^{\circ}$ acquisition of CT scans. To illustrate the potential of this device, we present results on the compression of a basalt glass obtained by $\mu \mathrm{CT}$, and diffraction/ scattering-CT imaging of the pressure-induced structural changes during $\mathrm{C}_{60}$ polymerization. On-going developments and further applications of this new device to other in situ tomography techniques are given as final prospects.

\section{Experimental method}

\subsection{The rotating tomographic cell}

The Paris-Edinburgh (PE) cell is a portable large-volume press based on an opposed anvil geometry (Besson et al., 1992). Using an X-ray/neutron transparent gasket, this geometry offers a large window in the equatorial plane suitable for a wide range of in situ high-pressure/hightemperature measurements (Le Godec et al., 2009). However, in the traditional V7 type PE press the opening is limited to $68^{\circ}$ owing to the shadows of the four tie rods, which is not suitable for CT experiments as the entire sample needs to be imaged. To overcome this limitation we designed a rotating module inspired from the stress cell developed by Bromiley et al. (2009). A detailed mechanical description of the so-called RoToPEc (rotational and tomography Paris-Edinburgh cell) module is given by Philippe et al. (2016). In brief, the two opposed anvils can rotate independently under load, with no limitation in the rotation angle, through two sets of gear reducers and thrust bearings located at the end of each anvil (Fig. 1). The accurate rotation of the anvils is monitored by stepper motors and encoders, with a precision of $0.02^{\circ}$. This allows the collection of data at small angular steps over $180^{\circ}$ rotation required for a complete $3 \mathrm{D}$ tomographic reconstruction. At full power, the motors enable rotation of the anvils under load within less than $10 \mathrm{~min}$ for a complete CT scan. Additionally, independent and controlled rotation of each anvil provides operation in shearing (one anvil rotates while the other is stationary) or in deformation (both anvils rotate in opposite direction) mode achieving large deformation rates $\gamma>2$ at speeds of $10^{-4}-10^{-7} \mathrm{~s}^{-1}$. The RoToPEc module fits the 450 ton frame of the V7 type PE press and therefore can cover a large pressure/temperature $(P-T)$ range 


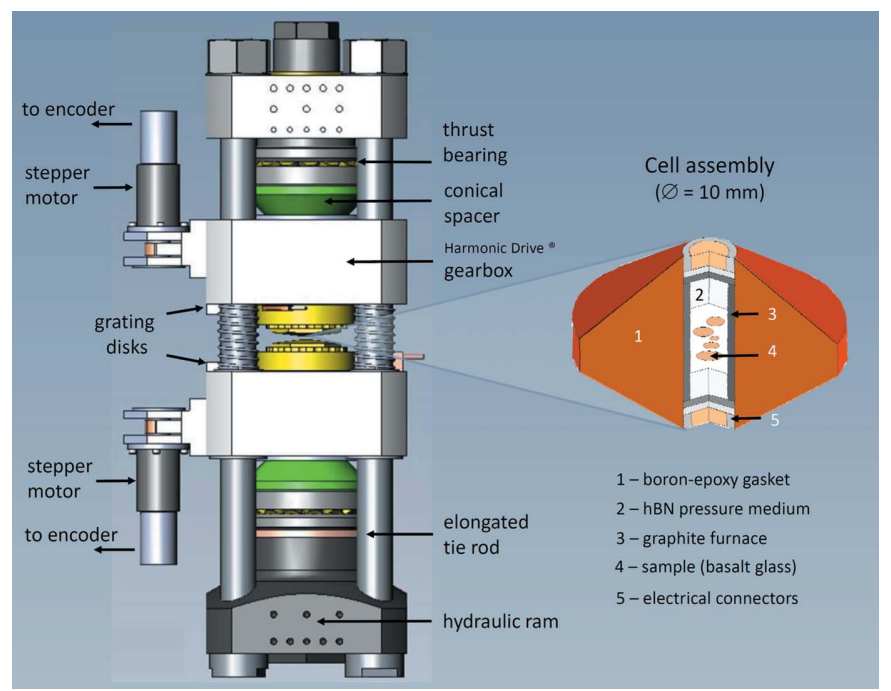

Figure 1

3D view of the 450 ton V7 Paris-Edinburgh press with the RoToPEc module, and details of the cell assembly used for in situ X-ray measurements.

(15 GPa/1500 $\mathrm{K}$ with sintered diamond anvils, and $10 \mathrm{GPa} /$ $2500 \mathrm{~K}$ with tungsten carbide anvils) and host large-volume samples (several $\mathrm{mm}^{3}$ ). Finally, the apparatus is compatible with a Sollers slit system, a multi-channel collimator (Mezouar et al., 2002; Morard et al., 2011) that is required in the diffraction mode to reduce the background scattering signal from the sample environment.

The sample assembly is essentially the same as the original PE press, using a boron epoxy transmitting medium and a graphite furnace. A poly ether-ether-ketone (PEEK) ring is added around the gasket to limit the lateral extrusion of the chamber during compression, as it can be a source of motion artifacts in the tomographic reconstruction. This containment ring also helps to hold a large X-ray window between the anvils suitable for full-field imaging mode in $\mu \mathrm{CT}$, and provides a homogeneous background for white-field corrections (see \$2.2.1). The oil pressure in the ram is generated by a computercontrolled hydraulic ('syringe') pump with a capacity of 3000 bar, while temperature is adjusted by varying the electrical power delivered to the furnace and controlled by a Eurotherm $\mathbb{R}$ regulation. This ensures a stability of $P-T$ conditions over the CT scan collection that can last several hours. Such a compact large-volume press set-up has a total mass of only $200 \mathrm{~kg}$. It can be easily disassembled/ assembled for easier transport, and its integration on a beamline only requires $x-y-z$ translations and a rotation $(\omega)$ stage for micro-positioning of the sample along the beam. To date, the RoToPEc device has been successfully used at the ID27 beamline of ESRF, the PSICHE beamline of Synchrotron SOLEIL, and the I12 station of Diamond Light Source.

\subsection{Data acquisition and tomographic reconstruction procedure}

2.2.1. X-ray absorption tomography at the PSICHE beamline of SOLEIL. The $\mu \mathrm{CT}$ measurements were performed on the white-beam station of the PSICHE beamline at SOLEIL (St Aubin, France). The configuration of the RoToPEc cell, $\mathrm{X}$-ray imaging and diffraction setups are presented in Fig. 2 [a detailed description of the beamline can be found by King et al. (2016)]. High-energy photons ranging continuously from 15 to $80 \mathrm{keV}$ are produced with an in-vacuum wiggler, and are reflected using an iridium X-ray mirror (angle $1.8 \mathrm{mrad}$, critical energy $\sim 46 \mathrm{keV}$ ) and subsequently filtered by a $1200 \mu \mathrm{m}$-thick copper foil to produce a beam spectrum with an average energy of $\sim 45 \mathrm{keV}$. The maximal size of the X-ray beam is $2.5 \mathrm{~mm}$ and $4.5 \mathrm{~mm}$ in the vertical and horizontal directions, respectively, defined by the entrance slits. The absorption contrasts within the sample are converted into visible light by a single-crystal LuAG phosphor, $90 \mu \mathrm{m}$ thick, set in the transmitted beam on the downstream side of the sample. A $45^{\circ}$ mirror reflects the visible contrast into the objective lens of a Hamamatsu ORCA Flash4.0 sCMOS camera, giving an effective pixel size of $2.45 \mu \mathrm{m}$. A conventional energy-dispersive X-ray (EDX) diffraction technique was used to determine pressure during experiments. For this purpose, the EDX signal from an internal pressure standard was collected using a Ge solid-state detector with necessary collimating optics on the diffraction side (Fig. 2).

To collect the $\mu \mathrm{CT}$ data the sample was rotated through $180^{\circ}$ by moving the anvils in unison with a speed of $0.15^{\circ} \mathrm{s}^{-1}$, while acquiring a continuous series of images. The complete
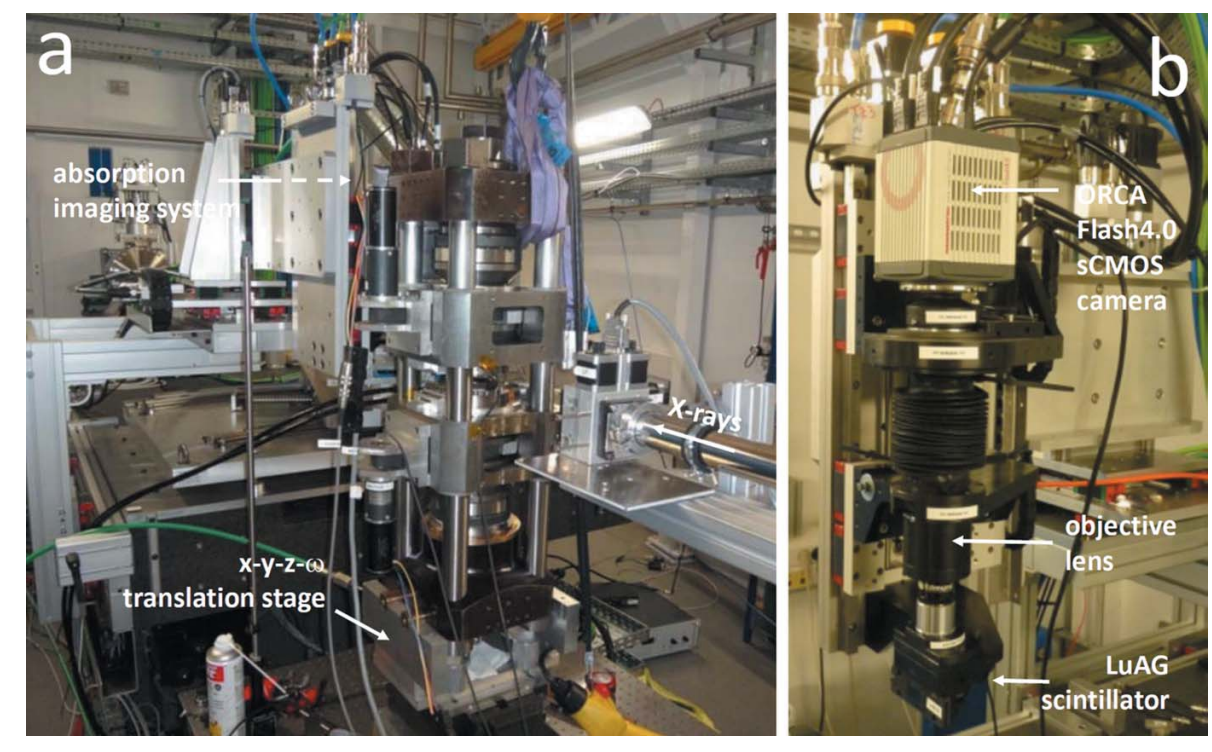

Figure 2

(a) Photograph of the RoToPEc device installed at the white end-beam station of the PSICHE beamline (Synchrotron SOLEIL), and $(b)$ details of the X-ray absorption imaging system. 
dataset consisted of 1400 radiographs with an angular resolution between frames of $\sim 0.13^{\circ}$ collected over $1200 \mathrm{~s}$. Flatfield images were collected immediately before the first image (at $0^{\circ}$ rotation) and after the last image (at $180^{\circ}$ rotation) by moving the press laterally about $\sim 5 \mathrm{~mm}$ so the X-ray beam passed only through the boron epoxy gasket and PEEK containment ring. The average of these two images was used as the white-field correction intended to remove non-uniformities in the X-ray beam intensity, imperfections in the LuAG scintillator and lens, and defects in the camera sensor. A darkfield image was also recorded with the beam off to account for thermal noise of the camera. The discrete angular interval between successive radiographs coupled with the resolution of the imaging system determine the voxel resolution of the reconstructed volume. In the present study, two-dimensional unbinned images with pixels of $2.45 \mu \mathrm{m} \times 2.45 \mu \mathrm{m}$ resulted in a voxel size of $7 \mu \mathrm{m}$ for $0.13^{\circ}$ angular steps.

The tomographic reconstruction involved a pre-processing stage where both the dark-field and the white-field images were subtracted from the raw images. Radiographs were then processed by the classical Radon transform and filtered backprojection algorithms to reconstruct slices of the sample perpendicular to the rotation axis. Filtered backprojection was performed using the PyHST2 software package (Mirone et al., 2014). Finally, the reconstructed slices were stacked to produce a $3 \mathrm{D}$ image of attenuation. The tomographic dataset was uploaded in Blob3D for visualization and further quantification analysis (see §3.1).

2.2.2. X-ray diffraction/scattering tomography at ID27 beamline of ESRF. The DSCT measurements were made at the ID27 beamline of ESRF (Grenoble, France) (Álvarez-Murga et al., 2012). Fig. 3 presents the experimental set-up for X-ray diffraction tomography experiments in the RoToPEc device; a more general description of the beamline is given by Mezouar et al. (2005). A Si-111 double-crystal monochromator is used to select the energy of the incoming beam at $33 \mathrm{keV}$. Using Kirkpatrick-Baez mirrors, the beam is focused

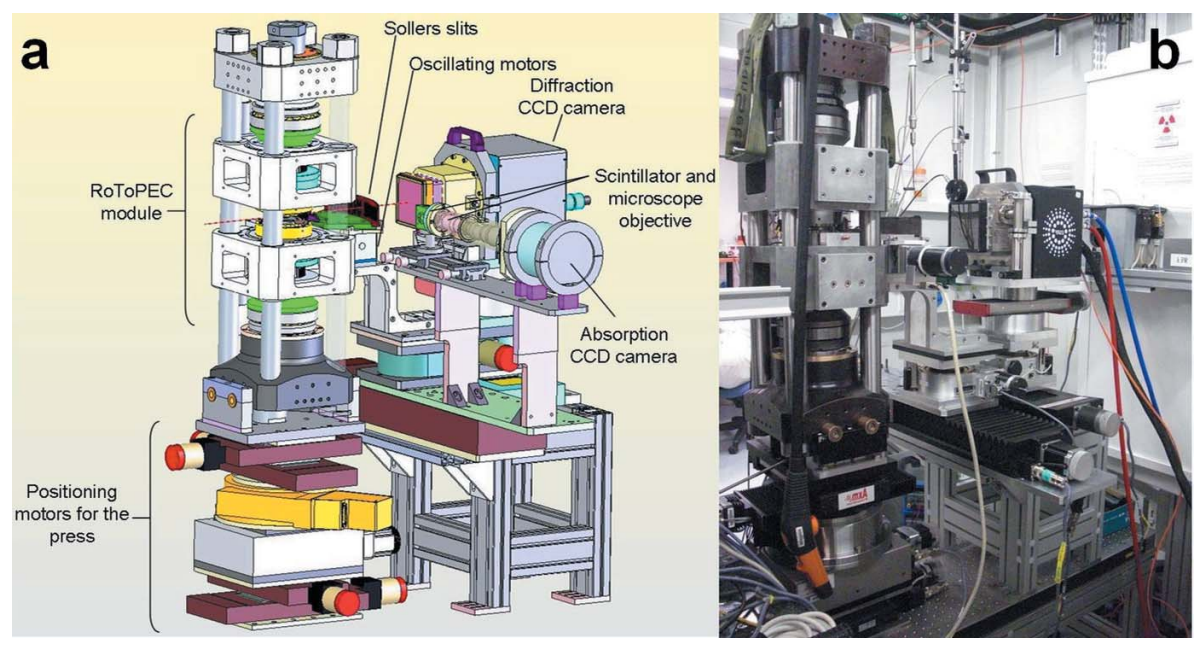

Figure 3

Schematic drawing $(a)$ and photograph $(b)$ of the micro-tomography setup installed at beamline ID27 of ESRF. down to a FWHM size of $2.4 \mu \mathrm{m} \times 2.0 \mu \mathrm{m}$ at the sample location. The press is installed on $x-y-z-\omega$ positioning motors. An ionization chamber is placed before the sample to monitor the incoming flux $\left(I_{0}\right)$ during acquisition for image normalization. A fast-readout low-noise (FReLoN) CCD camera (Labiche et al., 2007), with $2048 \times 2048$ pixel resolution, is used to collect the diffracted two-dimensional patterns in transmission geometry at a sample-detector distance of $370 \mathrm{~mm}$. A multichannel collimator, i.e. Sollers slits, is located in between the sample and detector to reduce the scattering signal from the sample environment. The beamline also offers the opportunity to perform simultaneously full-field imaging for $\mu \mathrm{CT}$ reconstructions. In this mode, KB mirrors are removed to produce a wide $4 \mathrm{~mm} \times 2 \mathrm{~mm}$ monochromatic illumination; Sollers slits are translated away from the transmitted beam and replaced by a radiography imaging set-up. Details of this imaging set-up are given by Perrillat et al. (2010).

In a DSCT experiment the sample is rotated $180^{\circ}$ around the $\omega$ axis and a set of diffraction patterns are collected for different positions of the sample along the $y$ axis, perpendicular to the X-ray beam. The complete dataset for the reconstruction of a single slice of the sample consists of several thousands of two-dimensional scattered diagrams, i.e. $N y$ translation steps $\times N \omega$ rotational steps. The use of Sollers slits currently limits the minimum exposure time for the acquisition of one X-ray diffraction (XRD) pattern to $10 \mathrm{~s}$ (the time required for the oscillation of the slits to cover the full $2 \theta$ range) leading to collection times of several hours for a complete DSCT scan. As a result of beam time limitation, the probed volume is restricted to a diameter of $0.5-1.5 \mathrm{~mm}$ with $\Delta y$ and $\Delta \omega$ steps in the range $5-30 \mu \mathrm{m}$ and $1-5^{\circ}$, respectively. This long acquisition time also stresses the need to maintain stable high $P-T$ conditions for several hours.

In the DSCT reconstruction procedure (Bleuet et al., 2008; Álvarez-Murga et al., 2011), the two-dimensional XRD patterns are first azimuthally integrated using Fit $2 D$ in sequence mode (Hammersley et al., 1996) to produce a set of one-dimensional patterns. The integrated intensity profiles are then plotted as a function of angular projection $\omega$ to construct a so-called sinogram $S(y, \omega)$. Different regions of interest corresponding to specific Bragg peaks $(i)$ are selected for the reconstruction. Therefore, specific sinograms $S(y, \omega)_{i}$ can be constructed for each crystalline/amorphous phase. The reconstruction of a two-dimensional virtual slice $(x, y)_{i}$ from a set of one-dimensional angular projections $S(y, \omega)_{i}$ is achieved using the standard algebraic reconstruction algorithm (Natterer \& Wübbeling, 2001; Bleuet et al., 2008). During this process, the images are also normalized for changes of direct beam intensity $\left(I_{0}\right)$ during data 
Table 1

Chemical composition of the natural basaltic glass (wt\%).

Analysis was performed using the X-ray fluorescence technique. The total iron content was calculated as $\mathrm{Fe}_{2} \mathrm{O}_{3}$. The low lost on ignition (LOI) value is shown for a lack of hydrothermal alteration of the glass sample.

\begin{tabular}{llllllllllll}
\hline $\mathrm{SiO}_{2}$ & $\mathrm{Al}_{2} \mathrm{O}_{3}$ & $\mathrm{TiO}_{2}$ & $\mathrm{MgO}$ & $\mathrm{Fe}_{2} \mathrm{O}_{3}$ & $\mathrm{CaO}$ & $\mathrm{Na}_{2} \mathrm{O}$ & $\mathrm{MnO}$ & $\mathrm{K}_{2} \mathrm{O}$ & $\mathrm{P}_{2} \mathrm{O}_{5}$ & LOI & Total \\
\hline 49.87 & 14.95 & 1.41 & 7.92 & 10.43 & 12.04 & 2.61 & 0.17 & 0.12 & 0.13 & -0.45 & 99.65 \\
\hline
\end{tabular}

fitted to a third-order Birch-Murnaghan EoS using the EoSFit7.0 software (Angel et al., 2014). The best fit yields an isothermal bulk modulus $K_{0}=69.3 \mathrm{GPa}$ and a pressure derivative $K^{\prime}=3.6$, or alternatively $K_{0}=68.6 \mathrm{GPa}$ when $K^{\prime}$ is fixed to $K^{\prime}=4$. These values are in good agreement with previous Brillouin spectroscopy measurements that reported $K_{\mathrm{s}}=67.2 \mathrm{GPa}$ on a natural basalt (Malfait et collection. The reconstruction provides an isotropic resolution of the two-dimensional-slice $(x, y)_{i}$ and the slice thickness is equal to the vertical beam size. A major asset of DSCT is the possibility to extract the scattering/diffraction from a selected area of the tomography image. This is done by recovering a one-dimensional intensity profile along the $2 \theta$ axis for any given voxel of the image, discarding the contributions of the surrounding voxels. These selective patterns can be used for any further structural analysis (i.e. Rietveld refinement).

\section{Tests results}

\subsection{Compressibility of a basaltic glass from $\mu \mathrm{CT}$ volume measurements}

$\mathrm{X}$-ray absorption computed tomography is one of the most direct methods for determining the compressibility of noncrystalline materials by measuring the physical change in volume as pressure varies. In this study, we investigated the compression behavior of a natural basaltic glass. Constraints on the equation of state $(\mathrm{EoS})$ of silicate glasses are indeed crucial for modeling magmas in planetary interiors. Fragments of a basaltic glass from the East Pacific Ridge [similar to the material used by Perrillat $e t$ al. (2006)] were placed in an airdriven sphere grinder to produce spheroid samples a few hundreds of micrometers in diameter. The chemical composition of this glass as measured by X-ray fluorescence is listed in Table 1. Five of these glass beads were embedded in hexagonal boron nitride (h-BN) powder and loaded into a $10 \mathrm{~mm}$ boron-epoxy gasket (Fig. 4). Pressure was increased at $300 \mathrm{~K}$ up to $4.4 \mathrm{GPa}$ in $\sim 0.6 \mathrm{GPa}$ steps, and calibrated from the diffraction lines of h-BN using the EoS parameters of Le Godec et al. (2000). At each pressure interval a $\mu$ CT image was recorded following the procedure described previously (\$2.2.1). Fig. 4 presents a representative $\mathrm{X}$-ray radiograph and the $3 \mathrm{D}$ reconstructed volume at $0.9 \mathrm{GPa} / 300 \mathrm{~K}$.

Images of the reconstructed slices through the sample were uploaded into Blob3D (Ketcham, 2005) to determine the volume of each glass bead as a function of pressure. All images were first median filtered for noise attenuation, and subsequently segmented following a grayscale threshold analysis. This segmentation steps enabled the isolation of voxels with particular intensity values, and their assignment to the 'basaltic glass' phase. Post-segmentation morphological operations, i.e. erosion/dilation, filing or smoothing, were not performed. We then determined the relative volume change $V / V_{0}$ of each glass bead as a function of pressure to produce the compression curve presented in Fig. 5. These data were al., 2011) and $K_{\mathrm{s}}=70 \mathrm{GPa}$ for an Iceland basalt (Liu \& Lin, 2014). (Note that with the difference between the constant pressure and the constant volume heat capacities for silicate glasses being small we can reasonably assume that $K_{\mathrm{s}} \simeq K_{0}$.) This good agreement with previous elastic data results from the ability of $\mu \mathrm{CT}$ to resolve small volume changes of $\leq 2 \%$, making $\mu \mathrm{CT}$ under high pressure a new method to determine

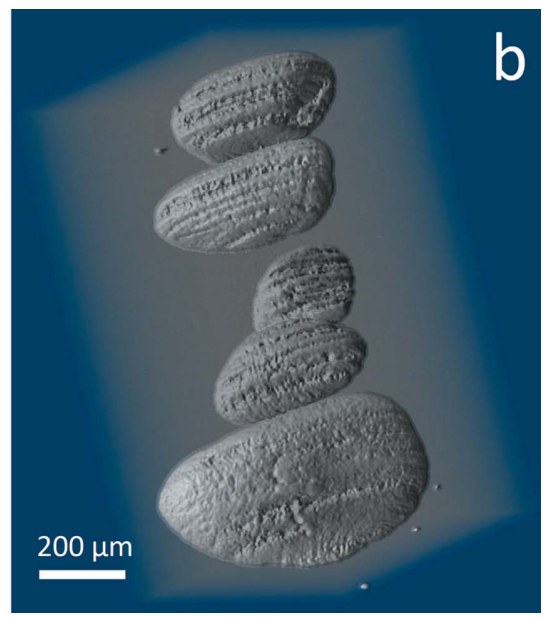

Figure 4

(a) Representative raw X-ray radiograph of the five glass blobs embedded in h-BN pressure medium, and $(b) 3 \mathrm{D}$ rendered volume from the $\mu \mathrm{CT}$ scan recorded at $0.9 \mathrm{GPa} / 300 \mathrm{~K}$.

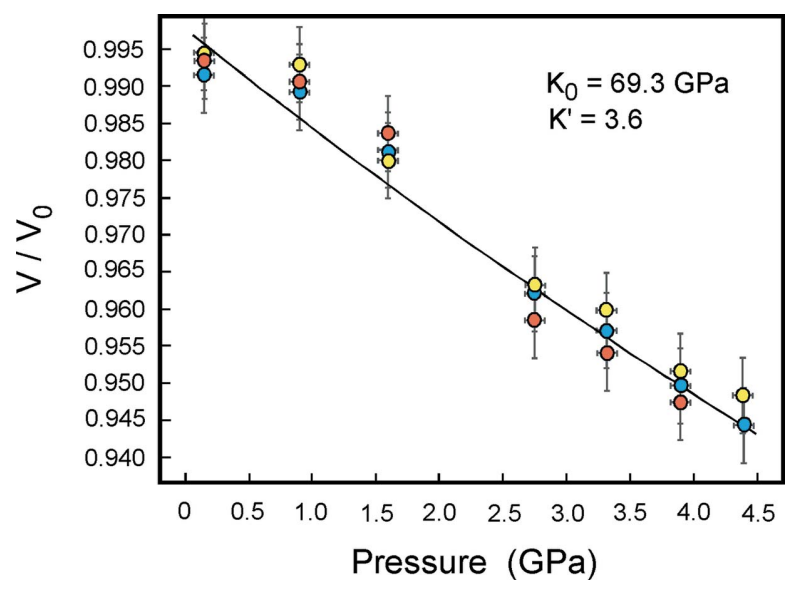

Figure 5

Room-temperature compression curve of MORB glass determined from the relative volume change of each glass beads extracted from $B l o b 3 D^{\circledR}$. Symbols with different colors correspond to $V / V_{0}$ measurements performed on different blobs. The best fit using a third-order BirchMurnaghan EoS is shown as the solid curve, and gives $K_{0}=69.3 \mathrm{GPa}$

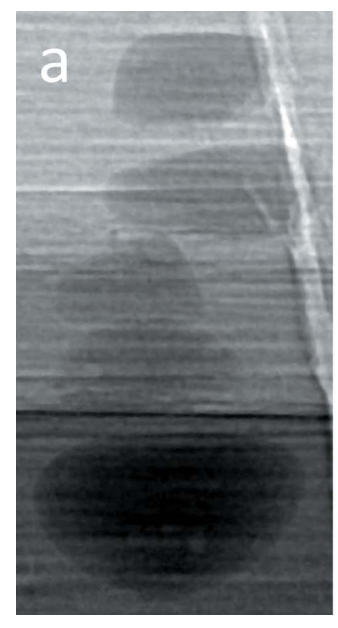
with $K^{\prime}=3.6$ 
the EoS of amorphous compounds even for materials with relatively high incompressibility like silicate glasses. Although the present work only reports data at ambient temperature, subsequent measurements have been performed on $\mathrm{FeS}+$ olivine mixtures at $3 \mathrm{GPa}$ and $1900 \mathrm{~K}$ (Philippe et al., 2016) showing the capability of simultaneous high-temperature and high-pressure $\mu \mathrm{CT}$ imaging.

\section{2. $\mathrm{C}_{60}$ polymerization processes under extreme $\boldsymbol{P}-\boldsymbol{T}$ conditions from 3D XRD tomography}

To demonstrate the potential of diffraction/scattering computed tomography for investigation of crystalline compounds with similar atomic densities and compositions, we investigated in situ the pressure-induced polymerization process of $\mathrm{C}_{60}$ molecular solid. The initial stages of polymerization, i.e. the formation of dimers and trimers, are crucial for the synthesis of the high-order polymers and disordered phases (Álvarez-Murga \& Hodeau, 2015). However, the identification of dimers and trimers among other polymer products remains a difficult task due to (i) the lack of in situ XRD studies and spatially resolved structural studies, (ii) the highly disordered dimer-states showing poorly resolved diffraction peaks and (iii) the coexistence of several lowdensity polytypes (e.g $\mathrm{C}_{60}$ monomer, trimers or $1 \mathrm{D}-2 \mathrm{D}$ polymers). Here we use the high-resolution structural probe of DSCT to follow the in situ structural evolution of $\mathrm{C}_{60}$ during polymerization.

High-purity $\mathrm{C}_{60}$ powder (Sigma-Aldrich, $99.9 \%$ ) was filled into a $0.9 \mathrm{~mm}$ h-BN capsule, placed inside a $3.5 \mathrm{~mm}$ cylindrical graphite furnace, encapsulated within a $10 \mathrm{~mm}$ boron-epoxy gasket. A PEEK containment ring was used to restrict the extrusion of the gasket during the tomographic measurements. The sample was cold-compressed at $2.4 \mathrm{GPa}$, as determined from the $d$-spacing of the (002) h-BN reflection. At beamline ID27 of ESRF, a monochromatic $33 \mathrm{keV}(\lambda=0.3738 \AA)$ pencil beam of $2.4 \mu \mathrm{m} \times 2.0 \mu \mathrm{m}$ was used to scan the sample along a $1.35 \mathrm{~mm}$ profile. The DSCT measurements consisted of a total of $23502 \mathrm{D}$-XRD patterns, i.e. 47 rotation steps $\left(\Delta \omega=4^{\circ}\right) \times$ 50 translation steps $(\Delta y=27 \mu \mathrm{m}) \times 1$ slice, acquired in a collection time of $8 \mathrm{~h}$.

Fig. 6 shows the 2D-DSCT reconstructed slices for $d$ spacing/ $2 \theta$ of (111), (200), (220), (311) and (222) $\mathrm{C}_{60}$ reflections and (002) reflection from the h-BN container. The reliability of the reconstruction is demonstrated by the similarity of the rendered images, even when using the extremely weak (200) reflection. Moreover, the diameter of the sample chamber determined from the 2D slices is 905.5 (2) $\mu \mathrm{m}$, in good agreement with the initial loading. The reconstructed images exhibit a 'spotty' aspect as a consequence of the noncontinuous intensity of the Debye-Scherrer rings. This may be related either to a grain preferential orientation from the coldcompression or to a large grain size relative to the beam size. Nevertheless, the distribution of the (111), (200), (220), (311) and (222) $\mathrm{C}_{60}$ intensities seems to fill all the gasket, as if these reflections all belong to the same phase and are homogeneously distributed. The diffraction lines are consistent with

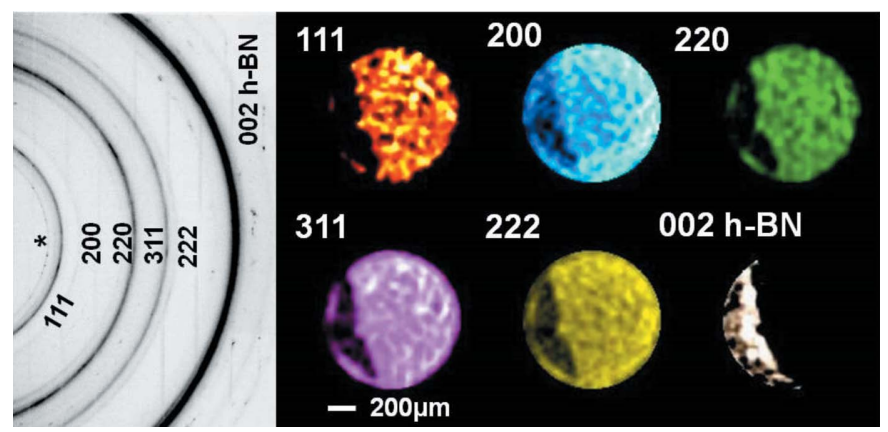

Figure 6

2D X-ray diffraction pattern and 2D reconstructed slices from $d$-spacing/ $2 \theta$ of $111,200,311$ and $222 \mathrm{C}_{60}$ reflections and 002 reflection from the h-BN container. Other reflections from the sample environment are marked *. Voxel resolution is $27.44 \mu \mathrm{m} \times 27.44 \mu \mathrm{m} \times 0.0078^{\circ}(x \times$ $y \times 2 \theta)$.

a fully dimerized $\mathrm{C}_{60}$ as shown by the presence of the (200) reflection peak which is missing in the face-centered cubic phase, and the broadening and shift of all peaks to larger angles (Davydov et al., 1999). The average lattice parameters at $2.4 \mathrm{GPa} / 300 \mathrm{~K}$ calculated from $1 \mathrm{D}$ projected patterns are $a=$ $13.55 \AA(220,311,222), a=13.61 \AA$ (111) and $a=13.42 \AA$ (200). No traces of remaining $\mathrm{C}_{60}$ monomer or high-order polymers were detected during posterior analysis of the onedimensional profiles extracted from the reconstructed volume (within the detection limits the probe $\sim 0.1 \%$ volume). In contrast, a large amount of h-BN was detected inside the reaction chamber, probably caused by the deformation of the cell during high-pressure extrusion. Additional DSCT scans have been acquired during two dedicated runs at $1.5 \mathrm{GPa} /$ $700 \mathrm{~K}$ and $4.4 \mathrm{GPa} / 600 \mathrm{~K}$ (Álvarez-Murga, 2012). The 3DXRD images are still under reconstruction, and the implications on the $\mathrm{C}_{60}$ reaction diagram will be published in a dedicated paper.

In classical DSCT the length of the pencil beam along the sample can degrade the $2 \theta$ resolution because the diffraction volume is not a point source. This results in a broadening of diffraction lines that may severely affect large objects. In the present experiment, the Sollers slits define a discrete diffraction volume (i.e. diffraction lozenge) that leads to a significant improvement of peak sharpness and makes the interpretation of rendered images easier as fewer peaks overlap. The use of Sollers slits, though increasing the acquisition time, is thus particularly akin to the present scientific case where the $\mathrm{C}_{60}$ dimerization is characterized, among others, by a broadening of diffraction lines.

\section{Conclusion and perspectives}

Here, we have reported the development of new sets-ups for tomography measurements under extreme conditions. We have demonstrated the potential of the 'RoToPEc' to perform $\mathrm{X}$-ray absorption based tomography for $3 \mathrm{D}$ volume rendering, and determined the compressibility of a natural basalt glass as an illustration of the ability of this new technique to resolve volume changes $\leq 2 \%$ under compression or heating. We also 
reported the first implementation of synchrotron X-ray diffraction/scattering micro-tomography under pressure, by investigating the structural changes during the polymerization of $\mathrm{C}_{60}$ molecules. The DSCT gives access to micrometer scale 3D phase-selective maps and allows the retrieval of local structure information like crystallite size, micro-strain, preferential orientations and defects, and is particularly appropriate for the characterization of complex multi-phase and multi-scale materials. Although not discussed here, the RoToPEc offers also the opportunity to shear the sample by differential rotation of the top and bottom anvils (Philippe et al., 2016). Combined with tomography, this enables the 3D textural and morphological evolution under strain to be monitored which is extremely useful in studying the mechanical behavior of multiphase materials. Further developments of this device include the use of sintered diamond anvils and/or toroidal gaskets (Kono et al., 2015) to enlarge the $\mathrm{X}$-ray window available for $\mathrm{CT}$ imaging that is presently affected by the reduction of the gap between the WC anvils at the highest pressures. On-going efforts are also concentrated on the speed-up of data collection in the goal to record timeresolved CT scans, and exploring dynamic processes like phase transformation kinetics or fluid/melt migration.

In conclusion, the combination of high pressure, high temperature and X-ray microtomography provides a novel 3D probe to obtain insight into the evolution of microstructure, phase distribution, strain-state and volume changes of materials under extreme conditions. This covers a wide range of potential applications in physics, chemistry, geology, earth, planetary and materials sciences. The RoToPEc device opens also new perspectives in neutron tomography though difficulties related to the gasket pressure transmitting medium (i.e. absorption) and the $P-T$ stability over acquisition time must be overcome.

\section{Acknowledgements}

We acknowledge the European Synchrotron Radiation Facility and Synchrotron SOLEIL for the allocation of beam time. We are grateful to S. Bauchau, M. Berg, G. Bromiley, G. Garbarino, J. P. Itié and S. Redfern for valuable inputs and discussions. This work was financially supported by the French 'Réseau Hautes Pressions', the Agence Nationale de la Recherche ANR-2011-BS08-018, a CNRS grant 'Instrumentation aux limites 2013', and the 'Institut National des Sciences de l'Univers'. MA acknowledges CONACYT grant 211996.

\section{References}

Álvarez-Murga, M. (2012). PhD thesis, Université Grenoble, France. Álvarez-Murga, M., Bleuet, P. \& Hodeau, J.-L. (2012). J. Appl. Cryst. 45, 1109-1124.

Álvarez-Murga, M., Bleuet, P., Marques, L., Lepoittevin, C., Boudet, N., Gabarino, G., Mezouar, M. \& Hodeau, J.-L. (2011). J. Appl. Cryst. 44, 163-171.

Álvarez-Murga, M. \& Hodeau, J. L. (2015). Carbon, 82, 381-407.
Angel, R. J., Gonzalez-Platas, J. \& Alvaro, M. (2014). Z. Kristallogr. 229, 405-419.

Baker, D. R., Brun, F., O'Shaughnessy, C., Mancini, L., Fife, J. L. \& Rivers, M. (2012). Nat. Commun. 3, 1135.

Banhart, J. (2008). Advanced Tomographic Methods in Materials Research and Engineering. Oxford University Press.

Bernard, D., Gendron, D., Heintz, J. M., Bordère, S. \& Etourneau, J. (2005). Acta Mater. 53, 121-128.

Besson, J. M., Nelmes, R. J., Hamel, G., Loveday, J. S., Weill, G. \& Hull, S. (1992). Physica B, 180-181, 907-910.

Bleuet, P., Welcomme, E., Dooryhée, E., Susini, J., Hodeau, J.-L. \& Walter, P. (2008). Nat. Mater. 7, 468-472.

Bromiley, G. D., Redfern, S. A. T., Le Godec, Y., Hamel, G. \& Klotz, S. (2009). High. Press. Res. 29, 306-316.

Davydov, V. A., Kashevarova, L. S., Rakhmanina, A. V., Agafonov, V. N., Allouchi, H., Céolin, R., Dzyabchenko, A. V., Senyavin, V. M., Szwarc, H., Tanaka, T. \& Komatsu, K. (1999). J. Phys. Chem. B, 103, 1800-1804.

Godec, Y. L., Martinez-Garcia, D., Mezouar, M., Syfosse, G., Itié, J. P. \& Besson, J. M. (2000). High. Press. Res. 17, 35-46.

Hammersley, A. P., Svensson, S. O., Hanfland, M., Fitch, A. N. \& Häusermann, D. (1996). High Press. Res. 14, 235-248.

Ketcham, R. A. (2005). Geosphere, 1, 32-41.

King, A., Guignot, N., Zerbino, P., Boulard, E., Desjardins, K., Bordessoule, M., Leclerq, N., Le, S., Renaud, G., Cerato, M., Bornert, M., Lenoir, N., Delzon, S., Perrillat, J. P., Legodec, Y. \& Itié, J. P. (2016). Rev. Sci. Instrum. 87, 093704.

Kono, Y., Kenney-Benson, C., Shibazaki, Y., Park, C., Wang, Y. \& Shen, G. (2015). Rev. Sci. Instrum. 86, 072207.

Labiche, J. C., Mathon, O., Pascarelli, S., Newton, M. A., Ferre, G. G., Curfs, C., Vaughan, G., Homs, A. \& Carreiras, D. F. (2007). Rev. Sci. Instrum. 78, 091301.

Le Godec, Y., Hamel, G., Solozhenko, V. L., Martinez-Garcia, D., Philippe, J., Hammouda, T., Mezouar, M., Crichton, W. A., Morard, G. \& Klotz, S. (2009). J. Synchrotron Rad. 16, 513-523.

Lesher, C. E., Wang, Y., Gaudio, S., Clark, A., Nishiyama, N. \& Rivers, M. (2009). Phys. Earth Planet. Inter. 174, 292-301.

Lin, Y., Zeng, Q., Yang, W. \& Mao, W. L. (2013). Appl. Phys. Lett. 103, 261909.

Liu, J. \& Lin, J. F. (2014). Geophys. Res. Lett. 41, 8832-8839.

Liu, H., Wang, L., Xiao, X., De Carlo, F., Feng, J., Mao, H. K. \& Hemley, R. J. (2008). Proc. Natl Acad. Sci. USA, 105, 1322913234.

Malfait, W. J., Sanchez-Valle, C., Ardia, P., Médard, E. \& Lerch, P. (2011). Am. Mineral. 96, 1402-1422.

Mezouar, M., Crichton, W. A., Bauchau, S., Thurel, F., Witsch, H., Torrecillas, F., Blattmann, G., Marion, P., Dabin, Y., Chavanne, J., Hignette, O., Morawe, C. \& Borel, C. (2005). J. Synchrotron Rad. 12, 659-664.

Mezouar, M., Faure, P., Crichton, W., Rambert, N., Sitaud, B., Bauchau, S. \& Blattmann, G. (2002). Rev. Sci. Instrum. 73, 3570.

Mirone, A., Brun, A., Gouillart, E., Tafforeau, P. \& Kieffer, J. (2014). Nucl. Instrum. Methods Phys. Res. B, 324, 41-48.

Morard, G., Mezouar, M., Bauchau, S., Álvarez-Murga, M., Hodeau, J. L. \& Garbarino, G. (2011). Rev. Sci. Instrum. 82, 023904.

Natterer, F. \& Wü bbeling, F. (2001). Mathematical Methods in Image Reconstruction. Monographs on Mathematical Modeling and Computation 5, p. 110. Philadelphia: SIAM.

Perrillat, J. P., Mezouar, M., Garbarino, G. \& Bauchau, S. (2010). High Press. Res. 30, 332-341.

Perrillat, J. P., Ricolleau, A., Daniel, I., Fiquet, G., Mezouar, M., Guignot, N. \& Cardon, H. (2006). Phys. Earth Planet. Inter. 157, 139-149.

Philippe, J., Le Godec, Y., Mezouar, M., Berg, M., Bromiley, G., Bergame, F., Perrillat, J. P., Alvarez-Murga, M., Morand, M., Atwood, R., King, A. \& Régnier, S. (2016). High. Press. Res. 36, 512-532. 
Poulsen, H. F., Jense, D. J. \& Vaughan, G. B. M. (2004). Mater. Res. 29, 166-169.

Rau, C., Somogyi, A. \& Simionovici, A. (2003). Nucl. Instrum. Methods Phys. Res. B, 200, 444-450.

Salvo, L., Cloetens, P., Maire, E., Zabler, S., Blandin, J. J., Buffière, J. Y., Ludwig, W., Boller, E., Bellet, D. \& Josserond, C. (2003). Nucl. Instrum. Methods Phys. Res. B, 200, 273-286.

Schroer, C. G. (2001). Appl. Phys. Lett. 79, 1912-1914.

Urakawa, S., Terasaki, H. P., Funakoshi, K., Uesugi, K. \& Yamamoto, S. (2010). J. Phys. Conf. Ser. 215, 012026.
Wang, Y., Lesher, C., Fiquet, G., Rivers, M. L., Nishiyama, N., Siebert, J., Roberts, J., Morard, G., Gaudio, S., Clark, A., Watson, H., Menguy, N. \& Guyot, F. (2011). Geosphere, 7, 40-53.

Wang, Y., Uchida, T., Westferro, F., Rivers, M. L., Nishiyama, N., Gebhardt, J., Lesher, C. E. \& Sutton, S. R. (2005). Rev. Sci. Instrum. 76, 073709.

Wang, J., Yang, W., Wang, S., Xiao, X., De Carlo, F., Liu, Y. \& Mao, W. L. (2012). J. Appl. Phys. 111, 112626.

Yu, T., Wang, Y. \& Rivers, M. (2016). Progr. Earth Planet. Sci. 3, 17. 